\title{
ENC(C) Review Questions
}

Section Editor: Heather McLellan, MEd, BN, RN, CEN, CFRN

Authors: Margaret Dymond, BSN, RN, ENC(C), Leanne Tyler, MR, RN, MHM, ENC(C)

1. Which of the following patients may be at more risk of developing acute tubular necrosis (ATN) and renal failure?

A) A 34-year-old female with pyelonephritis

B) A 34-year-old male with a UTI

C) A 34-year-old male with multiple trauma and hypotension

D) A 34-year-old male with unstable angina

2. The physician orders activated charcoal 50 grams PO for a patient with polypharmacy drug ingestion. The patient has a GCS of 8, B/P 100/70, HR 104, RR-12, SpO2 93\% on room air. Time of ingestion is unknown. What is the priority intervention?
A) Administer activated charcoal PO as ordered
B) Request an order to insert a gastric tube verses $\mathrm{PO}$
C) Insert a nasogastric tube as the risk of aspiration is lower
D) Inform the physician that the patient has a GCS of 8 and requires re-assessment and airway management prior to acti- vated charcoal administration.

3. EMS arrives with a 65-year-old female who fell down a flight of stairs and was found unresponsive. Her vital signs are $B / P 190 / 100$, heart rate 52, respiratory rate 10, GCS 8 and she has an oral airway in situ and unequal pupils. The vital signs and clinical assessment best describes:
A) Cushing's triad
B) Babinski reflex
C) Cushing's syndrome
D) Clonus reflex

4. Chest pain that is sudden in nature and severe is associated with:
A) Aortic dissection
B) Gastrointestinal reflux
C) Pneumonia
D) Acute coronary syndrome

5. A 24-year-old male arrives at the emergency department. He stumbles into the triage area, appears confused and disorientated, speech rambling, agitated, uncooperative. The patient is uncooperative with any attempt to get vital signs. The triage nurse recognizes a high priority is which of the following?
A) Call the police
B) Obtain the patient's blood glucose
C) Place the patient in a secure room and call security
D) Restrain the patient at triage to obtain vital signs

6. The physician is examining a patient's eye and decides an intraocular pressure is needed. What structure in the eye is this measuring?
A) Posterior chamber
B) Choroid
C) Anterior chamber
D) Lens

7. A patient has been diagnosed with a pulmonary embolism (PE). Lab results reveal a slightly elevated troponin. Which of the following is the most likely pathophysiologic mechanism for this result?

A) Increased right ventricular (RV) afterload

B) Plasmin degradation of fibrin

C) Increased RV myocardial stretch

D) Systemic activation of inflammatory mediators

8. A 24-year-old male has been bucked off a horse, landing on his back. He arrives complaining of right flank pain and hematuria. He denies abdominal pain. Which of the following rationales supports this finding?

A) Injuries to the genitourinary system always have hematuria

B) The kidneys are located in the peritoneal space

C) The patient may have a rib fracture

D) The kidneys are located in the retroperitoneal space

9. A five-year-old patient is admitted with severe fluid volume deficit due to gastroenteritis. His weight is $18 \mathrm{~kg}$. Which of the following would be an appropriate initial bolus for fluid resuscitation?
A) D5 $1 / 2 \mathrm{NS} 450 \mathrm{ml}$
B) NS $360 \mathrm{ml}$
C) LR $180 \mathrm{ml}$
D) $3 \% \mathrm{NS} 500 \mathrm{ml}$

10. An elderly patient presents to the ED with signs of acute stroke. While conducting the motor portion of a neurologic exam, you observe downward drift and pronation of the left forearm. This finding indicates which of the following?
A) Right cerebral hemisphere lesion
B) Left cerebral hemisphere lesion
C) Cerebellar incoordination
D) Dorsal column lesion

\section{Answer Key with Rationale}

1. Correct Answer: C

Rationale: Severe hypotension and poor perfusion to the kidney may cause ATN and renal failure. ATN occurs when there is a prolonged ischemic insult to the kidneys. Other conditions that may result in ATN are sepsis, any condition involving volume loss (e.g., GI bleeding) or a nephrotoxin (e.g., vancomycin). Most patients who have a UTI do not develop ATN. A young male with unstable angina would not be expected to develop ATN (Erdbruegger \& Okusa, 2020).

\section{Correct Answer: D}

Rationale: Activated charcoal is best administered within one hour of the ingestion. There still may be a potential clinical benefit after one hour. To avoid aspiration in a patient who has altered mental status is to secure the airway by intubating the patient. The patient should have a gastric tube inserted followed by 
activated charcoal administration via the gastric tube if ordered. Activated charcoal would be contraindicated if the ingestion was a hydrocarbon (gasoline, lamp oil), toxins poorly absorbed (metals-Iron, Lithium, toxic alcohols- methanol), or presence of an intestinal obstruction (Hendrickson \& Kusin, 2019).

\section{Correct Answer: A}

Rationale: Cushing's reflex (triad) is common in patients with acute brain injury with signs of increased ICP and a compensatory mechanism to preserve cerebral blood flow. The patient will have a widening pulse pressure (the difference between the systolic and diastolic blood pressure), a reflex bradycardia, and respiratory depression. Babinski reflex is an abnormal finding in adults and is fanning of the big toe when the foot is simulated. Cushing syndrome is reflective of a person taking a corticosteroid medication over time. Clonus occurs with interruption of the upper motor neurons. (Smith \& Amin-Haniaini, 2019; Fareedy \& Pathak, 2015).

\section{Answer: A}

Rationale: Pain that starts suddenly and is severe at onset is associated with aortic dissection, pneumothorax, and pulmonary embolism. Discomfort from an acute coronary syndrome typically starts gradually and may worsen with exertion. With stable angina, discomfort occurs only when activity creates an oxygen demand that outstrips supply limitations imposed by a fixed atherosclerotic lesion. This occurs at relatively predictable points and changes slowly over time. Unstable angina represents an abrupt change from baseline functioning, which may manifest as discomfort that begins at lower levels of exercise or at rest (Hollander \& Chase, 2020).

\section{Answer: B}

Rationale: Always consider a medical emergency may exist before a mental health condition. A chief complaint of confusion or isolated abnormal mental status implies that vital signs are stable and normal or near normal, which excludes many life-threatening conditions. Patients with abnormal behaviour are screened for hypoxia and hypoglycemia by bedside testing (Huff, 2019).

\section{Answer: C}

Rationale: The anterior chamber is the fluid-filled space inside the eye between the pupil and the cornea's innermost surface, the endothelium. Aqueous humour is the fluid that fills the anterior chamber. Hyphema and glaucoma are two main pathologies in this area. In hyphema, blood accumulates in the anterior chamber. In glaucoma, blockage of the canal of Schlemm prevents the normal outflow of aqueous humour, resulting in accumulation of fluid, increased intraocular pressure, and eventually blindness (Nolan-Kelley, 2020).

\section{Answer: A}

Rationale: In pulmonary embolism, troponin may be slightly elevated due to right heart strain (increased afterload); however, this result must be interpreted cautiously. Patients with pre-existing heart failure or renal failure may also have increased troponin levels (Rali et al., 2016, p. 134). D-dimer reflects the plasmin degradation of cross-linked fibrin in clots (products of thrombus degradation) (McCance \& Rote, 2019, p. 956). D-dimer may also be elevated in PE; although highly sensitive for clot formation, it has relatively low specificity for the diagnosis of PE (Rali et al., 2016, p. 134). In the setting of increased left and/or right ventricular myocardial stretch, such as in heart failure, brain natriuretic peptide (BNP) is released causing vasodilation and increased sodium and water loss via the kidneys (Lough, 2018, pp. 179-180). Lastly, C-reactive protein (CRP) is the most frequently used serum laboratory study to assess for the presence of systemic inflammatory mediators (Haynes 2018, p. 291).

\section{Answer: D}

Rationale: The kidneys are located in the retroperitoneal space, the anatomical space behind the peritoneum. They are situated at the level of the 12th thoracic vertebra to the 3rd lumbar vertebra and lie posterior to the stomach, spleen, colonic flexure, and small bowel (Bacidore, 2020, p. 467). The peritoneal space lies between the parietal and visceral peritoneum; it is a 'potential' space filled with a small amount of serous fluid, allowing the membranes to slide freely over each other without friction (Bacidore, 2020, p. 465). Injuries to the genitourinary system do not always result in hematuria. For example, mechanism of injury (MOI) and degree of bladder distention are key to suggesting potential for bladder injury. A pelvic fracture sustained in a motor vehicle collision, along with a full bladder, is more likely to result in hematuria than mild blunt force to the abdomen with an empty bladder (Bacidore, 2020, pp. 473-475).

\section{Answer: B}

Rationale: In the pediatric patient, fluid resuscitation requires rapid bolusing with $20 \mathrm{ml} / \mathrm{kg}$ of an isotonic crystalloid solution, as needed, until improvement is seen. NS is an isotonic solution; it is similar in osmolality and composition to body fluids (e.g., plasma), thus more fluid remains in intravascular space to expand intravascular volume. D5 $1 / 2$ NS (D5/0.45\% NS) is a hypertonic solution most appropriate for maintenance fluids; however, once the dextrose is metabolized, it becomes hypotonic. It provides some nutrients (e.g., glucose) to ensure nutritional intake. LR is an isotonic crystalloid solution; however, it is most often used post-operatively to buffer $\mathrm{pH}$ and replace electrolytes (e.g., calcium, potassium). $3 \%$ NS is a hypertonic solution used mainly in severe hyponatremia. It is not appropriate for fluid resuscitation due to risk of fluid overload, pulmonary edema, and central pontine myelinolysis (Khin, 2016, pp. 320321; Kuiper, 2020, p. 194)

\section{Answer: A}

Rationale: Pronator drift indicates abnormal function of the corticospinal tract (upper motor neurons) in the contralateral hemisphere. Cerebellar incoordination is seen when an arm tapped briskly downward, returns to its original position but overshoots and bounces. A lesion in the dorsal column is associated with a loss of proprioception (position sense) and is seen when an arm drifts up or laterally while the eyes are closed (Marsh \& Banasik, 2019). 


\section{About the authors}

Leanne Tyler, RN, MN, MHM, ENC(C)

Faculty, Course Developer - Critical Care/Emergency Nursing

Program, Centre for Professional Nursing Education, MacEwan

University

\section{REFERENCES}

Bacidore, V. (2020). Abdominal and genitourinary trauma. In V. Sweet \& A. Foley (Eds.), Sheehy's emergency nursing: Principles and practice (7th ed., pp. 465-476). Elsevier.

Erdbruegger, U., \& Okusa, M. D. (2020). Etiology and diagnosis of prerenal disease and acute tubular necrosis in acute kidney injury in adults. UpToDate. https://www.uptodate.com/ contents/etiology-and-diagnosis-of-prerenal-disease-and-acute-t ubular-necrosis-in-acute-kidney-injury-in-adults

Fareedy, S. B. \& Pathak, R. (2015). Ankle clonus. Clin Case Rep, 3(6), 520. https://doi.org/10.1002/ccr3.251

Haynes, A. (2018). Cardiovascular disorders. In L. M. Urden, K. M. Stacy, \& M. E. Lough (Eds.), Critical care nursing: Diagnosis and management (8th ed., pp. 290-358). Elsevier.

Hendrickson, R. G., \& Kusin, S. (2019). Gastrointestinal decontamination of the poisoned patient. UpToDate. https:// www.uptodate.com/contents/gastrointestinal-decontaminationof-the-poisoned-patient

Hollander, J. E., \& Chase, M. (2020). Evaluation of the adult with chest pain in the emergency department. UpToDate. https://www. uptodate.com/contents/evaluation-of-the-adult-with-chest-painin-the-emergenc $y$-department

Huff, J. S. (2019). Evaluation of abnormal behavior in the emergency department. UpToDate. https://www.uptodate.com/contents/ evaluation-of-abnormal-behavior-in-the-emergency-department

Khin, A. (2016). Fluid and electrolytes: Balance and disturbance. In P. Paul, R. A. Day, \& B. Williams (Eds.), Brunner \& Suddarth's Canadian textbook of medical-surgical nursing (3rd. ed., pp. 282333). Wolters Kluwer.

Kuiper, B. (2020). Fluids, fluid replacement, electrolytes, and vascular access. In V. Sweet \& A. Foley (Eds.), Sheehy's emergency nursing: Principles and practice (7th ed., pp. 193-204). Elsevier.
Margaret Dymond, RN, BSN, ENC(C), DCS

Clinical Nurse Educator, Emergency Department, University of

Alberta Hospital, Stollery Childrens Hospital, Mazankowski Heart

Institute
Ledford, L. (2020). Neurologic emergencies. In V. Sweet \& A. Foley (Eds.), Sheehy's emergency nursing: Principles and practice (7th ed., pp. 249-259). Elsevier.

Lough, M. E. (2018). Cardiovascular anatomy and physiology. In L. M. Urden, K. M. Stacy, \& M. E. Lough (Eds.), Critical care nursing: Diagnosis and management (8th ed., pp. 161-183). Elsevier.

Marsh, J.D. \& Banasik, J.L. (2019). Acute disorders of brain function. In J.L. Banasik \& L.C. Copstead (Eds.), Pathophysiology (6th ed., pp. 892-913). Elsevier.

McCance, K. L., \& Rote, N. S. (2019). Alterations of erythrocyte, platelet, and hemostatic function. In K. L. McCance \& S. E. Huether (Eds.), Pathophysiology: The biologic basis for disease in adults and children (8th ed., pp. 926-962). Elsevier.

Nolan-Kelley, L. (2020). Ocular emergencies. In V. Sweet \& A. Foley (Eds.), Sheehy's emergency nursing: Principles and practice (7th ed., pp. 365-376). Elsevier.

Rali, P., Gandhi, V., \& Malik, K. (2016). Pulmonary embolism. Critical Care Nursing Quarterly, 39(2), 131-138. https://doi. org/10.1097/CNQ.0000000000000106

Stacy, K. M. (2018). Neurologic anatomy and physiology. In L. M. Urden, K. M. Stacy, \& M. E. Lough (Eds.), Critical care nursing: Diagnosis and management (8th ed., pp. 520-549). Elsevier.

Smith, E. R., \& Amin-Hanjani, S. (2019). Evaluation and management of elevated intracranial pressure in adults. UpToDate. https:// www.uptodate.com/contents/evaluation-and-management-ofelevated-intracranial-pr essure-in-adults

Woods, L. S. (2018). Facial and ocular emergencies. In V. Sweet (Ed.), Emergency nursing core curriculum (7th ed., 246-258). Elsevier. 